\title{
Turning a yield-stress calcite suspension into a shear-thickening one by tuning inter-particle friction
}

\author{
James A. Richards ${ }^{1}$ (1) . Rory E. O'Neill ${ }^{1}$. Wilson C. K. Poon ${ }^{1}$
}

Received: 28 July 2020 / Revised: 24 September 2020 / Accepted: 28 September 2020 / Published online: 13 November 2020 (C) The Author(s) 2020

\begin{abstract}
We show that a suspension of non-Brownian calcite particles in glycerol-water mixtures can be tuned continuously from being a yield-stress suspension to a shear-thickening suspension-without a measurable yield stress—by the addition of various surfactants. We interpret our results within a recent theoretical framework that models the rheological effects of stress-dependent constraints on inter-particle motion. Bare calcite particle suspensions are found to have finite yield stresses. In these suspensions, frictional contacts that constrain inter-particle sliding form at an infinitesimal applied stress and remain thereafter, while adhesive bonds that constrain inter-particle rotation are broken as the applied stress increases. Adding surfactants reduces the yield stress of such suspensions. We show that, contrary to the case of surfactant added to colloidal suspensions, this effect in non-Brownian suspensions is attributable to the emergence of a finite onset stress for the formation of frictional contacts. Our data suggest that the magnitude of this onset stress is set by the strength of surfactant adsorption to the particle surfaces, which therefore constitutes a new design principle for using surfactants to tune the rheology of formulations consisting of suspensions of adhesive non-Brownian particles.
\end{abstract}

Keywords Suspension $\cdot$ Yield stress $\cdot$ Shear thickening $\cdot$ Rheology $\cdot$ Calcite $\cdot$ Dispersants

\section{Introduction}

A transformation has recently occurred in our understanding of the rheology of suspensions of hard non-Brownian (nB) particles (size $\gtrsim 2 \mu \mathrm{m}$ ) with repulsive interactions. Such suspensions shear thicken: their viscosity increases with applied shear rate (Barnes 1989). It is now accepted that stress-driven contact formation plays the dominant role (Lin et al. 2015; Clavaud et al. 2017; Comtet et al. 2017). Particles in mechanical contact cannot freely slide past one another due to Coulomb friction (Seto et al. 2013) or other mechanisms (Hsu et al. 2018; James et al. 2018). This happens when the stabilising repulsive force between particles fails to keep them separated when the applied stress increases beyond a critical threshold, $\sigma^{*}$, the 'onset stress'. The close approach of particle surfaces switches on

James A. Richards

jamesrichards92@gmail.com

1 SUPA, School of Physics and Astronomy, University of Edinburgh, James Clerk Maxwell Building, Peter Guthrie Tait Road, Edinburgh, EH9 3FD, UK anti-sliding mechanisms (Wilson and Davis 2000; Wyart and Cates 2014). The additional particle motion needed on the local level to accommodate any given macroscopic strain leads to extra dissipation, so that the viscosity rises (Lerner et al. 2012).

Most industrial nB suspensions are not purely repulsive. Typically, at high enough volume fraction, $\phi$, there exists a yield stress, $\sigma_{y}$, below which there is no flow. Above $\sigma_{y}$ the suspension shear thins and the viscosity decreases to a limiting plateau value. Practical examples span diverse sectors, from suspensions of mineral powders (Zhou et al. 1995) and polymeric latices (Heymann et al. 2002) to coal slurries (Wildemuth and Williams 1985) and molten chocolate (Blanco et al. 2019), for which glass spheres with hydrophobic coating in water (Brown et al. 2010) may function as a generic model system. In such applications, it is important to be able to 'tune' the yield stress, for example in unset concrete. To allow pumping and filling of formwork, $\sigma_{y}$ must not be too high (Roussel 2007); in contrast during $3 \mathrm{D}$ printing of concrete, too low a $\sigma_{y}$ and the unset concrete will not hold the desired shape before setting (Mechtcherine et al. 2020). It is therefore important to understand the origin of the yield stress in $\mathrm{nB}$ suspensions. 
A finite suspension yield stress is typically traced back to residual van der Waals attraction between particles that are insufficiently stabilised (Bonn et al. 2017). Such particles can 'bond' so that above some critical $\phi \ll$ random close packing they form a stress-bearing network. To flow, a finite stress $\sigma>\sigma_{y}$ must be applied to break the bonds and fluidise the suspension. A classic way to tune $\sigma_{y}$ is by adding surfactants, variously known as dispersants, plasticisers or other sector-specific terms. These adsorb onto particle surfaces, increase the minimum separation, and so reduce attraction and $\sigma_{y}$.

This explanation undoubtedly applies to colloids, where Brownian motion drives aggregation, and has been used to explain yield stress in $\mathrm{nB}$ suspensions (Brown et al. 2010). To see that something may be amiss in the latter case, consider an aqueous suspension of calcite particles, as found in toothpastes, paints and paper coatings. Later, we show that a $\phi \approx 0.5$ suspension of this kind with particle dimension $d \approx 4 \mu \mathrm{m}$ has $\sigma_{y} \approx 10^{2} \mathrm{~Pa}$ under steady shear. Dimensional analysis of the colloidal picture suggests that $\sigma_{y} \sim U / d^{3}$, with the energy scale $U$ set by the van der Waals interaction. Taking $U \sim A d / 12 h$ for two spheres at surface separation $h$ with the Hamaker constant, $A$, of calcite in water (Bergström 1997), we find $h \sim 0.01 \AA$, far below the atomic scale. It is evident we are missing some vital physics.

The missing physics is particle contact, which, if attractive forces are present, can prevent rolling below a critical torque when the contacts are pinned (Heim et al. 1999; Estrada et al. 2011). Such adhesive contacts constrain interparticle rolling, just as frictional contacts prevent sliding. The effect of sliding and rolling constraints acting independent or together on viscosity has been explored in a 'constraint rheology' framework (Guy et al. 2018), in which there are two critical stress scales, the onset stress for making frictional contact, $\sigma^{*}$, and the strength of an adhesive contact, $\sigma_{a}$.

Below we review constraint rheology (Guy et al. 2018), present data for a model calcite suspension and then consider the data in the context of constraint rheology. The finite yield stress in the bare-particle system can be 'tuned away' by adding surfactants. Making sense of our observations using the constraint rheology framework allows us to interpret the role of different surfactants in such adhesive $\mathrm{nB}$ suspensions, which turns out to differ radically from how they act in Brownian suspensions.

\section{Constraint rheology of suspensions}

For suspensions of hard particles, the relative viscosity, $\eta_{r}$, is controlled by the proximity to the jamming volume fraction. The viscosity divergence at some jamming point,
$\phi_{J}$, is captured by the form of Krieger and Dougherty (1959),

$\eta_{r}=\eta / \eta_{s}=\left[1-\phi / \phi_{J}\right]^{-\ell}$,

with $\ell \gtrsim 2$ (Guy et al. 2015). At $\phi \geq \phi_{J}$, the system shear jams and fractures under deformation (Brown and Jaeger 2014; Dhar et al. 2020). In the Wyart and Cates (2014) (WC) model for shear thickening, $\phi_{J}$ depends on the stress-dependent fraction of frictional contacts,

$f(\sigma)=\exp \left[-\left(\sigma^{*} / \sigma\right)^{\beta}\right]$,

which increases at the onset stress, $\sigma^{*}$, from $f\left(\sigma \ll \sigma^{*}\right)=0$ to $f\left(\sigma \gg \sigma^{*}\right)=1$, with a rapidity set by $\beta$. The increasing fraction of frictional contacts lowers the jamming point via

$\phi_{J}=\phi_{\mu} f+\phi_{\mathrm{rcp}}(1-f)$,

from random close packing, $\phi_{J}(f=0)=\phi_{\text {rcp }}$ at $\sigma \ll$ $\sigma^{*}$, to a lower frictional jamming point, $\phi_{J}(f=1)=$ $\phi_{\mu}$, at $\sigma \gg \sigma *$, whose value depends on inter-particle friction (Silbert 2010). For a suspension at a fixed $\phi<\phi_{\mu}$, a $\phi_{J}(\sigma)$ decreasing with stress gives a viscosity that increases from a low-shear plateau, $\eta_{r}^{0}$, to a high-shear plateau, $\eta_{r}^{\infty}$ (Eq. 1), as observed.

Importantly, adhesive constraints that limit inter-particle rolling can also lower $\phi_{J}$ (Guy et al. 2018; Richards et al. 2020). These are broken above a critical torque, $M_{a}$, set by the attractive force between particles and a surface length scale that pins the contact (Heim et al. 1999). The fraction of adhesive contacts, $a$, decreases rapidly above a characteristic stress, $\sigma_{a} \sim M_{a} / d^{3}$, which we model by

$a(\sigma)=1-\exp \left[-\left(\sigma_{a} / \sigma\right)^{\kappa}\right]$,

where $\kappa$ controls how rapidly $a$ decreases from $a(\sigma \ll$ $\left.\sigma_{a}\right)=1$ to $a\left(\sigma \gg \sigma_{a}\right)=0$.

With adhesive constraints alone, jamming occurs at 'adhesive close packing', $\phi_{J}(a=1, f=0)=\phi_{\text {acp }}$. Since the number of constraints (two rolling degrees of freedom) is the same as that in a purely frictional system (two sliding degrees of freedom), we take $\phi_{\mathrm{acp}}=\phi_{\mu}$. If both constraints operate, jamming occurs at a lower concentration, 'adhesive loose packing', $\phi_{J}(a=1, f=1)=\phi_{\text {alp }}<\phi_{\text {acp }}$. This critical volume fraction, which is possibly related to rigidity percolation (Richards et al. 2020), is not yet precisely known; one simulation returns $\phi_{\mathrm{alp}} \approx 0.14$ (Liu et al. 2017). The various jamming points are summarised in Table 1 .

In any actual suspension, $0 \leq f\left(\sigma / \sigma^{*}\right), a\left(\sigma / \sigma^{*}\right) \leq$ 1 , and $\phi_{J}(\sigma)$ depends on the degree to which frictional/adhesive contacts are formed/broken by the applied stress. We use a phenomenological ansatz to extend Eq. 3:

$$
\begin{aligned}
\phi_{J}= & f\left[\phi_{\mathrm{alp}} a+\phi_{\mu}(1-a)\right]+ \\
& (1-f)\left[\phi_{\mathrm{acp}} a+\phi_{\mathrm{rcp}}(1-a)\right] .
\end{aligned}
$$


Table 1 Table of jamming volume fractions in decreasing order. For quasi-monodisperse hard spheres, $\phi_{\text {rcp }} \approx 0.64$ and $\phi_{\mu} \approx 0.55$ are well documented; one simulation suggests $\phi_{\text {alp }} \approx 0.14$

\begin{tabular}{lll}
\hline Frac. frictional contacts, $f$ & Frac. adhesive contacts, $a$ & $\phi_{J}$ \\
\hline 0 & 0 & $\phi_{\text {rcp }}$ \\
1 & 0 & $\phi_{\mu}$ \\
0 & 1 & $\phi_{\text {acp }}=\phi_{\mu}$ \\
1 & 1 & $\phi_{\text {alp }}$ \\
\hline
\end{tabular}

The rheology clearly depends on the ratio $\theta=\sigma_{a} / \sigma^{*}$. In a 'frictional suspension', $\theta \gg 1\left(\sigma^{*} \ll \sigma_{a}\right), f=1$ at all accessible stresses and the flow is always frictional. Adhesion stabilises frictional contact networks, so that the system can jam and a yield stress develops at some rather low $\phi_{\text {alp }}$. Increasing the stress on a jammed system releases proportionately more adhesive constraints, so that $\phi_{J}$ monotonically increases from $\phi_{\text {alp }}$ to $\phi_{\mu}$. This monotonic $\phi_{J}(\sigma)$ produces a corresponding monotonic shear-thinning $\eta_{r}(\sigma)$ (Eq. 1 and Fig. 1, solid (blue)).

In a 'lubricated suspension', $\theta \ll 1\left(\sigma^{*} \gg \sigma_{a}\right)$, increasing $\sigma$ beyond $\sigma_{a}$ rapidly releases rolling constraints while $f \approx 0$. Thus, $\phi_{J}$ increases from $\phi_{\text {acp }}$ (which we take to be $=\phi_{\mu}$ ) towards $\phi_{\text {rcp }}$, until $\sigma \rightarrow \sigma^{*}$ and frictional contacts start to form, whereupon $\phi_{J}$ decreases towards $\phi_{\mu}$. Such a non-monotonic $\phi_{J}(\sigma)$ gives rise to a corresponding non-monotonic $\eta_{r}(\sigma)$ (Eq. 1 and Fig. 1 (purple)). In this case, with no frictional contact network for adhesion to stabilise, the system cannot jam (and $\sigma_{y}=0$ ) below $\phi_{\text {acp }}$ $\left(=\phi_{\mu}\right.$ for us) (Richards et al. 2020).

Within this framework, then, we may 'tune' a suspension in the range $\phi_{\text {alp }}<\phi<\phi_{\text {acp }}=\phi_{\mu}$ from having a finite $\sigma_{y}$ to having essentially $\sigma_{y} \rightarrow 0$ - a many orders of magnitude change-by engineering a transition from the $\theta \gg 1$
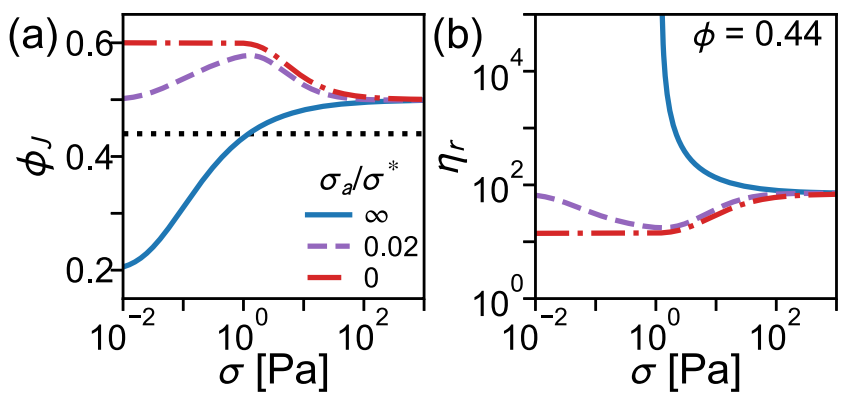

Fig. 1 Changing the ratio of the frictional onset stress to adhesive strength, $\theta=\sigma_{a} / \sigma^{*}$. a Stress-dependent jamming point, $\phi_{J}(\sigma)$, with decreasing $\theta$, Eqs. 2-5. Lines: solid (blue), yield-stress $\left(\sigma_{a}=0.1 \mathrm{~Pa}\right.$, $\left.\sigma^{*}=0 \mathrm{~Pa}\right)$; dashed (purple), thinning then thickening $\left(\sigma_{a}=0.1 \mathrm{~Pa}\right.$, $\left.\sigma^{*}=5 \mathrm{~Pa}\right)$; dot-dashed (red), shear-thickening $\left(\sigma_{a}=0 \mathrm{~Pa}, \sigma^{*}=5\right.$ $\mathrm{Pa}$ ); and, dotted (black), indicates a volume fraction of $\phi=0.44$. Other parameters: $\phi_{\mathrm{rcp}}=0.6, \phi_{\mu}=0.50, \phi_{\mathrm{alp}}=0.2, \beta=1, \kappa=0.6$. b Resultant flow curves at $\phi=0.44$ using Eq. $1(\ell=2), \sigma_{a} / \sigma^{*}$ as in (a) (frictional) regime to $\theta \ll 1$ (lubricated) regime. Below we show how to do this using surfactants in a calcite suspension.

\section{Materials and methods}

We studied ground calcium carbonate with a rhombohedral form (Eskal 500, KSL Staubtechnik GmbH (2007), 99\% purity, density $\rho_{p}=2.7 \mathrm{~g} / \mathrm{cm}^{3}, d_{50}=4 \mu \mathrm{m}$ ) (Fig. 2). Ground calcite is widely used as a filler in aqueous coatings to improve abrasion resistance and finish, or as a cheap extender. Powder was dispersed in glycerol-water mixtures using vortex and then high-shear mixing at $\phi \leq 0.45$, or manual stirring at $\phi>0.45$, until a smooth appearance was achieved. Using a glycerol-water mixture slows evaporation and sedimentation, both of which can prevent accurate rheology. The glycerol content was adjusted to access the maximum range of stresses in each sample $(0.1 \mathrm{~Pa} \lesssim \sigma \lesssim$ $400 \mathrm{~Pa}$ ). We checked that varying the amount of glycerol did not strongly change the rheology or qualitatively influence our conclusions.

We used three surfactants: polyacrylic acid (PAA); an alkyl-napthalene sulphonate condensate (ANS), Morwet D-425; and a polycarboxylate ether (PCE), Agrilan 755. PAA adsorbs to calcite forming a monolayer (Eriksson et al. 2007). Our PAA was a linear 5100 Da sodium salt (Sigma-Aldrich) with a 3-nm gyration radius (Reith et al. 2002). Commercial ANS is a highly polydisperse mixture of oligomers with branched and crosslinked chains (Piotte et al. 1995) often used as 'superplasticisers' in self-compacting concrete (Mehta 1999). The PCE is a comb co-polymer of polyethylene glycol grafted to a methacrylate-methylmethacrylate backbone.

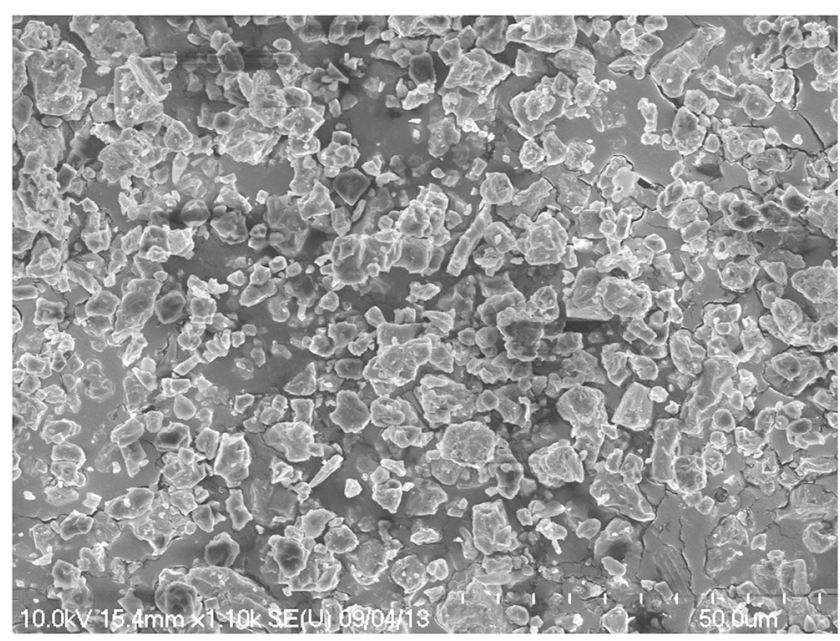

Fig. 2 Scanning electron micrograph of ground calcite, scale bar 50 $\mu \mathrm{m}$ 
Similar surfactants are known to stabilise calcite suspensions (Bossis et al. 2017).

Surfactants were dissolved into the glycerol-water mixture before powder incorporation at concentrations reported as weight percentages relative to the solid content (w/w\%). The main concentrations used were $0.05 \mathrm{w} / \mathrm{w} \%$ (PAA), $0.5 \mathrm{w} / \mathrm{w} \%$ (ANS) and $1.0 \mathrm{w} / \mathrm{w} \%$ (PCE), which were chosen as the concentration needed in each case before no further change was found in the rheology of a $\phi=0.44$ sample if more is added (see Appendix 1 for details).

Steady-state flow curves were measured using parallel plates (radius $R=20 \mathrm{~mm}$ and gap height $H=1 \mathrm{~mm}$ ) that were sandblasted or serrated to reduce slip. For suspensions without surfactant, controlled shear rate measurements were taken (TA Instruments ARES-G2, roughened plates). We report the rim shear rate, $\dot{\gamma}=\Omega R / H$ from the applied angular velocity, $\Omega$; the stress, $\sigma=\left(\mathcal{T} / 2 \pi R^{3}\right)(3+$ $\mathrm{d} \ln \mathcal{T} / \mathrm{d} \ln \Omega$ ) from the measured torque, $\mathcal{T}$; and hence the relative viscosity, $\eta_{r}=\sigma /\left(\dot{\gamma} \eta_{s}\right)$.

Samples were pre-sheared at high stress to remove memory of the loading history, before applying a single upsweep at 5 points per decade from the minimum shear rate, $\dot{\gamma}_{\min }=0.1 \mathrm{~s}^{-1}$, to sample fracture at $\sigma_{\max } \approx 400$ $\mathrm{Pa}$. At each point the longer of $\gamma=10$ or $t=10 \mathrm{~s}$ was accumulated with an average of the steady state taken. The minimum shear rate is set by the longest experiment limited by sedimentation below $\sigma_{\min }=\Delta \rho g d \approx 0.1$ $\mathrm{Pa}$. When strong shear thinning is observed we identify an experimental yield stress, $\sigma_{y}=\sigma\left(\dot{\gamma}_{\min }\right)$.

Measurements with surfactants used imposed stress (serrated plates, TA Instruments DHR-2 for ANS and PCE,
AR-2000 for PAA). After a $1 \mathrm{~Pa}$ pre-shear, 10 points per decade were measured between $\sigma_{\min }$ and fracture or inertial sample ejection, always ensuring reversibility below fracture. At each point, measurement followed a 5 s equilibration, but the total time per point was adjusted between samples to maximise the averaging time while still avoiding sedimentation. For PAA and PCE, the step time was $10 \mathrm{~s}$ for $\phi \leq 0.4,20 \mathrm{~s}$ for $0.45 \leq \phi \leq 0.49$ and $30 \mathrm{~s}$ for $\phi \geq 0.51$; for ANS, a single step time of $15 \mathrm{~s}$ was used. For systems where the shear rate may decrease with stress (discontinuous shear thickening), we report the apparent stress, $\sigma_{\text {app }}=2 \mathcal{T} / \pi R^{3}$.

\section{Results}

Suspensions of bare calcite particles in an 85 wt.\% glycerolwater mixture $\left(\eta_{s}=110 \mathrm{mPas}\right)$ show strong shear thinning at all measured $\phi$ (Fig. 3a), consistent with the presence of a yield stress below which flow ceases. At $\phi<0.40$, the viscosity decreases towards a high-shear plateau, $\eta_{r}^{\infty}$. At higher $\phi$ any plateau value is obscured by sample fracture (open symbols). At $\phi=0.50$ all flow may be due to fracture. In all cases, we take $\eta_{r}^{\infty}$ to be the viscosity at the highest stress before fracture.

The absence of shear thickening suggests that the system is always frictional. This is confirmed by fitting Eq. 1 to $\eta_{r}^{\infty}(\phi)$, giving divergence at $\phi_{\mu}=0.50(1)$ (with $\ell=$ 2.6(3)) (Fig. 3b). With $\sigma_{y} \approx \sigma_{\min }$ at $\phi=0.18$, it was not possible to measure a yield stress for lower $\phi$ without sedimentation; so, we take (as an upper bound) $\phi_{\mathrm{alp}}=0.18$.
Fig. 3 Steady-state rheology of bare calcite suspensions. a Relative viscosity vs stress, $\eta_{r}(\sigma)$, under imposed shear rate. Symbols, data at volume fraction, $\phi$ (legend). Shading (grey) outside measurable limits. b High-shear relative viscosity, $\eta_{r}^{\infty}(\phi)$. Symbols, $\eta_{r}$ before fracture; dotted line, $\eta_{r}^{\infty}=\left(1-\phi / \phi_{\mu}\right)^{-\ell}$, $\phi_{\mu}=0.50(1)$ (shading (grey)) and $l=2.6(3) \mathrm{c}$ Yield stress, $\sigma_{y}$, vs volume fraction. Symbols, yield stress from minimum shear rate, $\sigma_{y}=\sigma\left(\dot{\gamma}_{\min }=0.1 \mathrm{~s}^{-1}\right)$. Solid line, $\sigma_{y}$ with $\theta \rightarrow \infty$, from Eqs. 4 and 5 ( $f=1$ and $\left.\phi_{\text {alp }}=0.18\right)$, taking $\sigma_{a}=0.6 \mathrm{~Pa}$ and $\kappa=0.6$. Shaded (red), jammed; and unshaded, flowing
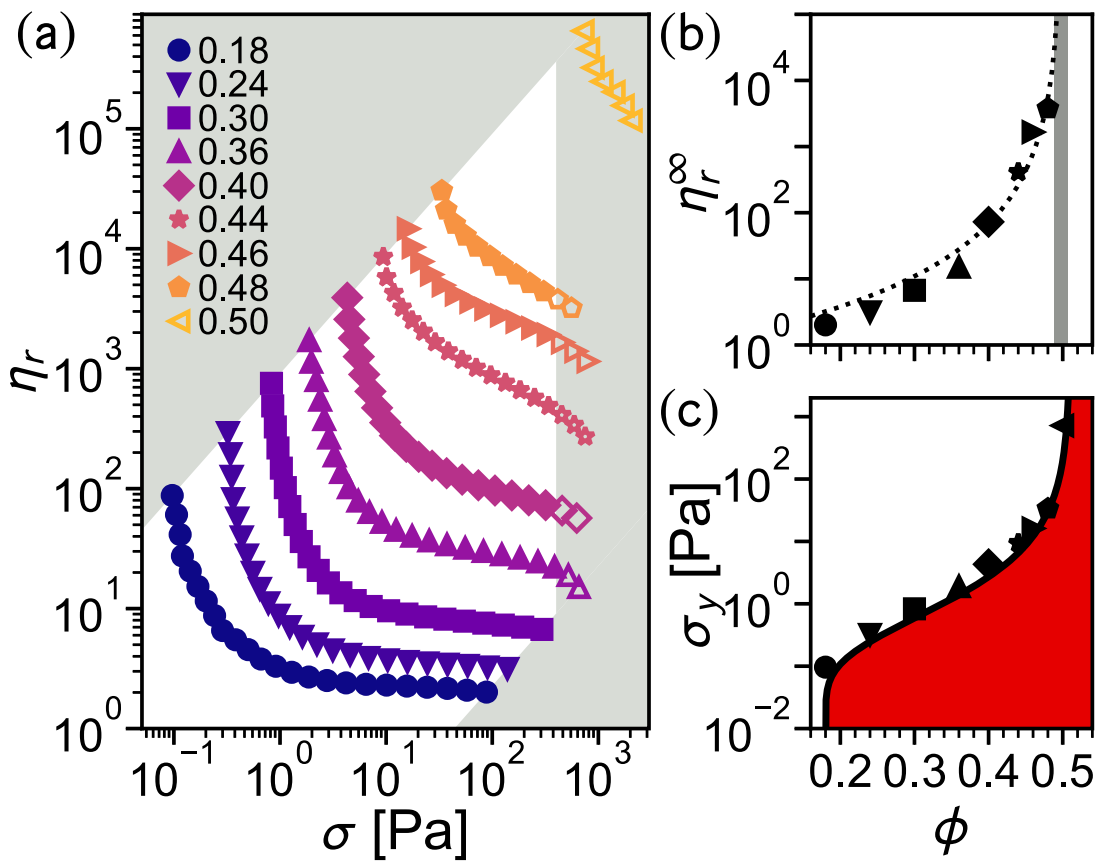
Using an adhesive strength of $\sigma_{a} \approx 0.6 \mathrm{~Pa}$ (and $\kappa=0.6$ ) in the constraint model of Eq. 5 with $f=1$ and Eq. 4 then predicts a $\phi$-dependence of $\sigma_{y}$ that accounts well for our observations (Fig. 3c).

Physically, Fig. $3 \mathrm{c}$ tells us that at low $\phi$ the suspension flows at all applied stresses. When $\phi$ reaches 0.18 , it becomes possible for adhesion to stabilise the frictional contact network enough for the system to jam. However, this adhesion-stabilised frictional network breaks above a finite applied stress, $\sigma_{y}$, and the system flows. As $\phi$ increases, the frictional network acquires additional stability, and more adhesive bonds need to be broken to fluidise the suspension: $\sigma_{y}$ increases. Eventually, upon reaching $\phi_{\mu}$, no adhesive stabilisation is needed and the frictional network is stable in its own right: the system is jammed at all stresses and $\sigma_{y} \rightarrow \infty$.

Thus, bare calcite particles in a glycerol-water mixture form a frictional suspension: little or no stress is needed to push the particles into frictional contact because $\sigma^{*} \rightarrow$ 0 . This onset stress can be made finite by introducing a repulsive barrier between particles, which then must be overcome to press particles into mechanical contact. This can be done, e.g. via electrostatic surface effects by modifying the dissolved ions (Al Mahrouqi et al. 2017). We will tune the repulsive barrier sterically using surfactants.

When PAA is added at a concentration where further addition causes no impact (which suggests monolayer coverage, Appendix 1) (Fig. 4a), the suspension no longer shows any evidence of a finite yield stress. Instead, the viscosity rises from a low-shear to a high-shear plateau at increasing stress, with the magnitude of the effect increasing with volume fraction (Fig. 5 (symbols)). This is classical shear thickening.

In detail, at $\phi \leq 0.47$, suspensions continuously thicken to a high-shear plateau, but for $\phi>0.47$ a plateau is not reached before fracture. At the highest measured $\phi=$ 0.52 , we see discontinuous shear thickening, indicated by $\mathrm{d} \ln \eta / \mathrm{d} \ln \sigma>1$ (dashed line). Fitting $\eta_{r}^{\infty}(\phi)$ to Eq. 1, Fig. 5 (inset (red)) gives the frictional jamming point $\phi_{J}=$ $\phi_{\mu}=0.49$ (1) (with $\ell=2.2(2)$ ), the same as the high stress $\phi_{J}$ for bare calcite suspensions to within error.

The absence of any observable shear thinning implies that $\sigma_{a} \rightarrow 0$. The observation of shear thickening means that, instead, $\sigma^{*}$ is now finite: a repulsive interaction must be overcome to press particles into mechanical contact, so that $\theta \rightarrow 0$. The removal of adhesion in the low-stress limit can be evidenced independently of shear rheology by studying sedimentation in dilute samples prepared by rigorous dispersal under high shear, followed by slow mixing. PAA-coated particles sediment slower than bare particles. This suggests that the former remain well dispersed, so that they are repulsively stabilised. By contrast, suspensions without PAA must contain aggregates; these will have been formed by adhesive particles being driven into contact during low-stress mixing (see Appendix 2 for details). With this stabilising repulsive interaction, the viscosity should diverge only at random close packing at $\sigma \rightarrow 0$. Fitting $\eta_{r}^{0}(\phi)$ to Eq. 1 (Fig. 5, inset
Fig. 4 Effect of surfactant concentration. a Variation of polyacrylic acid (PAA) sodium salt. Symbols, relative viscosity as a function of stress, $\eta_{r}(\sigma)$, at a volume fraction, $\phi=0.44$, in a $50 \mathrm{wt} \%$ glycerol-water mixture with PAA concentration weight percentage relative to calcite (w/w\%), legend. b Alkyl-naphthalene sulphonate condensate sodium salt (ANS) variation. c Polycarboxylate ether (PCE) variation
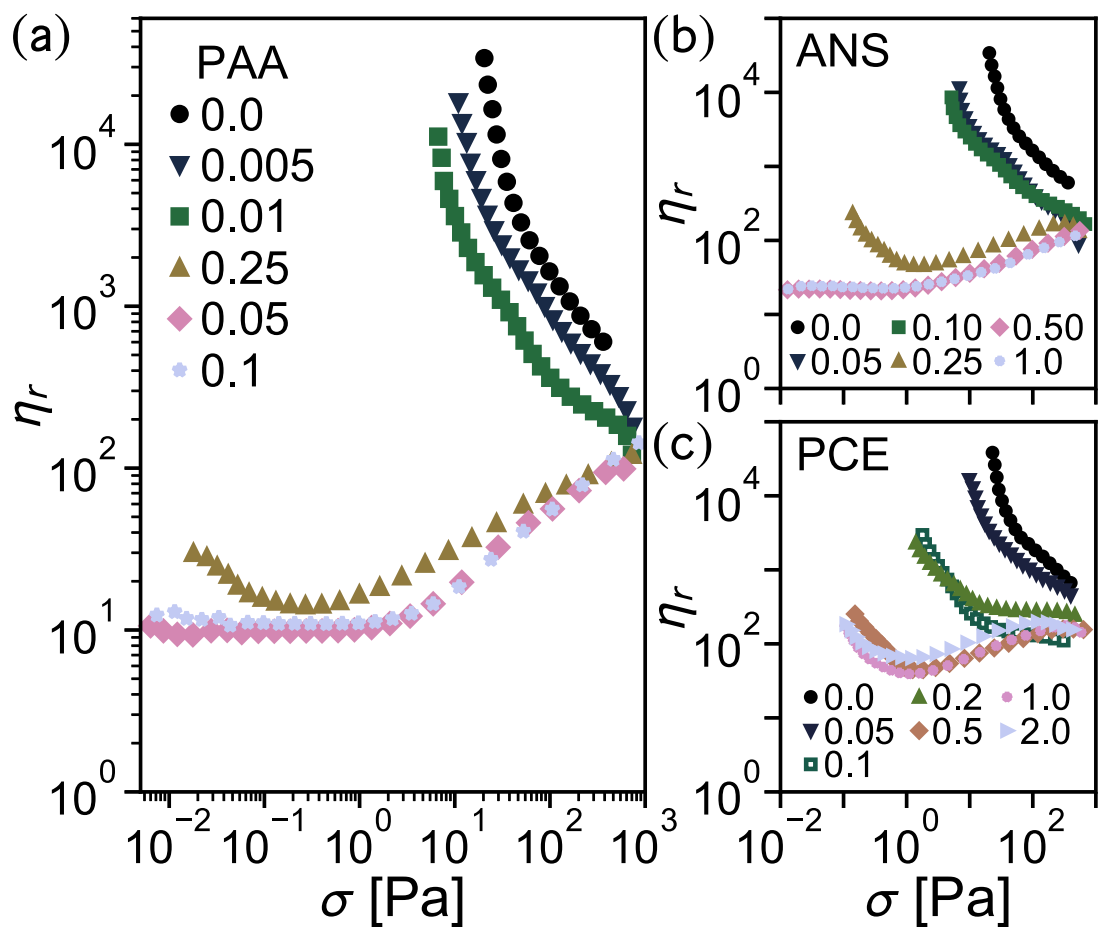


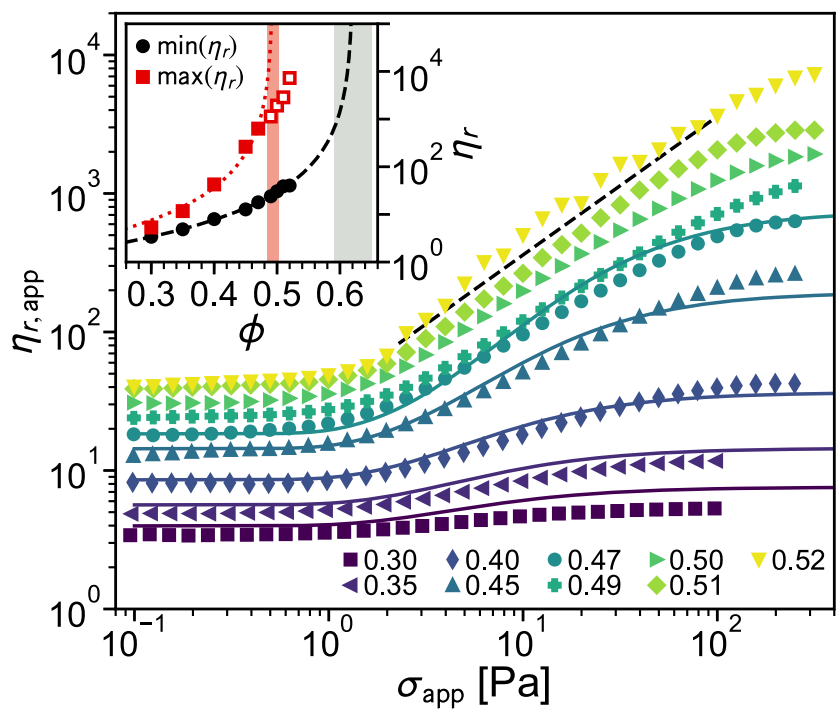

Fig. 5 Flow curves of polyacrylic acid (PAA) stabilised calcite suspensions in $70 \mathrm{wt} \%$ glycerol-water mixture, $\eta_{s}=22.5 \mathrm{mPas}$. Symbols: apparent relative viscosity vs stress, $\eta_{r \text {, app }}\left(\sigma_{\text {app }}\right)$, at volume fraction, $\phi$ (legend). Dashed (black) line, DST (slope $=1)$. Solid lines, WC model $(\theta \rightarrow 0)$ fit to $\phi \leq 0.47$, onset stress $\sigma^{*}=3.0$ (2) $\mathrm{Pa}(\beta=$ 1.02). Inset: plateau viscosity with volume fraction. Squares (red): high-shear viscosity from $\max \left(\eta_{r}\right)$; circles (black), low-shear viscosity from $\min \left(\eta_{r}\right)$. Dotted (red) line, fit of Eq. 1, to find $\phi_{\mathrm{J}}=0.49(1)$ (shading (red)) and $\ell=2.2(2)$. For $\min \left(\eta_{r}\right)$ a pre-factor, $A=0.8(1)$, is included in Eq. 1 (dashed (black) line), to give $\phi_{\mathrm{J}}=0.62(3)$ (shading (grey))

(black)), we find that, indeed, $\phi_{J}=0.62(3)$ (with $\ell=$ $2.2(4)$ ), consistent with the value of $\phi_{J}=0.60$ determined separately using a powder compaction test (DS/EN 1097-4:2008). Using our two fitted values of $\phi_{\text {rcp }}, \phi_{\mu}$ and $\ell=2.2$, from the high-shear viscosity divergence, we fit Eq. 2 to our data to find $\sigma^{*}=3.0$ (2) $\mathrm{Pa}$ (and $\beta=1.02$ ).

For ANS, again using a concentration where further addition causes no further change (Fig. 4b), a similar transition from a yield stress fluid to a shear-thickening suspension is seen (Fig. 6 (symbols)). Repeating the same analysis procedure, we find $\phi_{\mathrm{rcp}}=0.59(1)$ and $\phi_{\mu}=$ 0.499(3) (Fig. 6 (inset)), consistent with the PAA data. Again, the flow curves suggest that $\sigma_{a} \rightarrow 0$, and fitting Eq. 2 to the data now gives $\sigma^{*}=1.0(1) \mathrm{Pa}$, so that, again $\theta \rightarrow 0$.

The effect of the third surfactant, PCE at a concentration where further addition has no impact (Fig. 4c), is similar at moderate to high stresses, $\sigma \gtrsim 1 \mathrm{~Pa}$ (Fig. 7 (symbols)). We see continuous shear thickening to a plateau at $\phi \leq 0.47$ and to fracture at higher concentrations. On the other hand, we now see shear thinning at $\sigma \lesssim 1 \mathrm{~Pa}$, with the appearance of a small yield stress at the highest solid volume fractions, $\sigma_{y} \approx 0.2 \mathrm{~Pa}$ at $\phi=0.51$. This value is, however, negligible compared to that of the bare calcite suspension at this volume fraction $(\gtrsim 400 \mathrm{~Pa})$.

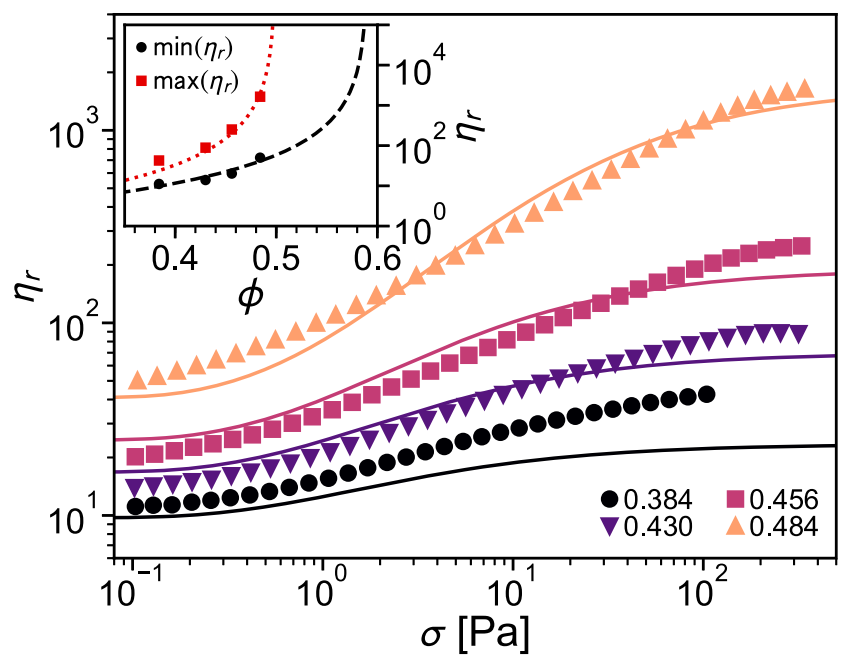

Fig. 6 Flow curves for alkyl-naphthalene sulphonate condensate (ANS) stabilised calcite suspensions in 85 wt.\% glycerol-water mixture, $\eta_{s}=110 \mathrm{mPas}$. Symbols: relative viscosity vs stress, $\eta_{r}(\sigma)$, at volume fraction, $\phi$ (legend); lines, WC model $(\theta \rightarrow 0)$ fit, $\sigma^{*}=1.0(1)$ $\mathrm{Pa}(\beta=0.67)$. Inset: limiting $\phi_{J}$ used in WC model. Symbols: squares (red), $\max \left(\eta_{r}\right)$, high-shear viscosity; circles (black), $\min \left(\eta_{r}\right)$, lowshear viscosity. Lines: dotted (red), fit of Eq. 1 to $\max \left(\eta_{r}\right)$ with $\ell=2.2$ fixed from Fig. 5 to find $\phi_{\mathrm{J}}=0.499(3)$; dashed (black), fit to $\min \left(\eta_{r}\right)$ to find $\phi_{\mathrm{J}}=0.59(1)$

Fitting $\eta_{r}^{\infty}(\phi)$ to Eq. 1 gives same frictional jamming point, $\phi_{\mu}=0.49(1)(l=2.3)$, as before. Fixing $\phi_{\mathrm{rcp}}=$ $0.62, \phi_{\mathrm{alp}}=0.18, \beta=0.67$ and $\kappa=0.6$ from previous fittings, we find that the constraint model can give a reasonable account of the observed trends using $\sigma_{a}=0.3$ $\mathrm{Pa}$ and $\sigma^{*}=3 \mathrm{~Pa}$ (Fig. 7 (dashed lines)), so that $\theta \approx 0.1$.

\section{Discussion and conclusions}

In the constraint rheology framework, the effect of adding surfactants is a matter of tuning the relative magnitudes of the two relevant characteristic stress scales, $\sigma^{*}$, beyond which sliding constraints form rapidly, and $\sigma_{a}$, beyond which rolling constraints break rapidly. The values of $\left(\sigma^{*}, \sigma_{a}\right)$ we have deduced (Table 2) should be taken as no more than order of magnitude estimates: varying the procedure used in inferring them from data would have given different results; but this does not alter the qualitative picture.

Dispersing bare $\mathrm{nB}$ calcite particles in a glycerol-water mixture gives suspensions in which a finite yield stress emerges at $\phi \gtrsim 0.18$ before jamming at all stresses at $\phi \gtrsim$ 0.5 , the frictional jamming point, $\phi_{\mu}$. The yield stress is due to adhesion of strength $\sigma_{a} \sim 0.6 \mathrm{~Pa}$ stabilising inter-particle frictional contacts. The presence of adhesion suggests there is van der Waals attraction (Liberto et al. 2019) and that the magnitude of electrostatic repulsion, controlled by the concentration of dissolved lattice ions (Al Mahrouqi et al. 2017), is insufficient to stabilise the particles. To 


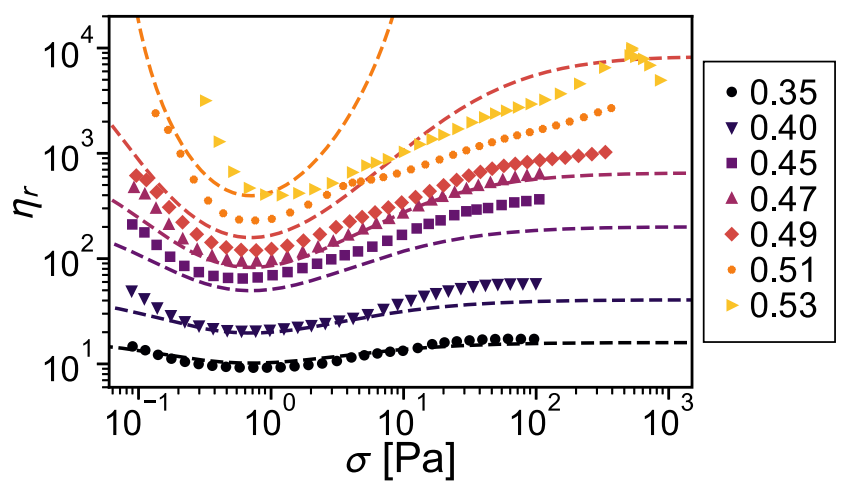

Fig. 7 Flow curves of polycarboxylate ether (PCE) stabilised ground calcite in $50 \mathrm{wt} . \%$ glycerol-water mixture, $\eta_{s}=6 \mathrm{mPas}$. (a) Relative viscosity vs stress, $\eta_{r}(\sigma)$. Symbols, data at volume fraction, $\phi$ (legend); dashed lines, Eqs. 1-5 with representative parameters, see text

constrain rolling, adhesion acts together with a pinning surface topography (Dominik and Tielens 1995). Thus, the facets of ground calcite particles (Fig. 2), which can inhibit rolling (Estrada et al. 2011) may enhance the strength of this constraint. In our coarse-grained model such shape effects are subsumed within an effective characteristic adhesive stress, $\sigma_{a}$.

For bare particles frictional contacts form at infinitesimal applied stress, i.e. the onset stress, $\sigma^{*}$, is vanishingly small. Adding any of the three surfactants confers a finite $\sigma^{*}$ of order $\sim 10^{0} \mathrm{~Pa}$. The difference between the surfactants is that while adding PAA and ANS reduces the adhesive strength to being immeasurably small, $\sigma_{a} \rightarrow 0$, adding PCE only decreases this stress scale by a factor of 2. Notably, this modest reduction in the adhesive strength still results in a large drop in the yield stress at, e.g., $\phi=0.40$ from 4 Pa to $\ll 0.1 \mathrm{~Pa}$, a factor of 40 (at least). With a large onset stress, $\theta \ll 1$, there is now no frictional contact network for adhesion to stabilise-frictional contacts are the primary determinants of the ability of the suspension to withstand finite applied stress.

Our PCE data (Fig. 7) resemble those for hydropho bised glass spheres in water (Brown et al. 2010). Brown et al. suggest that adding surfactant 'eliminates ... clustering

Table 2 Estimates of characteristic stresses in calcite suspensions without and with surfactants. For each system: frictional onset stress, $\sigma^{*}$; adhesive strength, $\sigma_{a}$; stress scale ratio, $\theta$; yield stress, $\sigma_{y}$, at $\phi=0.40 ;$ and the fitted frictional jamming point, $\phi_{\mu}$

\begin{tabular}{llllll}
\hline Surfactant & $\sigma^{*}(\mathrm{~Pa})$ & $\sigma_{a}(\mathrm{~Pa})$ & $\theta=\frac{\sigma_{a}}{\sigma^{*}}$ & $\begin{array}{l}\sigma_{y}(\mathrm{~Pa}) \text { at } \\
\phi=0.40\end{array}$ & $\phi_{\mu}$ \\
\hline None & $\rightarrow 0$ & 0.6 & $\rightarrow \infty$ & 4 & $0.50(1)$ \\
PAA & 3 & $\rightarrow 0$ & $\rightarrow 0$ & $\rightarrow 0$ & $0.49(1)$ \\
ANS & 1 & $\rightarrow 0$ & $\rightarrow 0$ & $\rightarrow 0$ & 0.50 \\
PCE & 3 & 0.3 & 0.1 & $\ll 0.1$ & 0.49 \\
\hline
\end{tabular}

with its associated yield stress and reveals a region of underlying shear thickening'. Instead, our data indicate the converse: that surfactants reduce $\sigma_{y}$ in adhesive $\mathrm{nB}$ suspensions primarily by imparting a finite onset stress below which there can be no frictional network for interparticle adhesion to stabilise.

Strikingly, the concentration at which the high-shear viscosity diverges for all three systems with surfactants occurs at $\phi \approx 0.50$ within experimental uncertainties, which is the frictional jamming point of the bare calcite suspension. At high applied stress, particles in the systems with surfactant additives interact as if they were bare. We interpret this as follows. Adding the amount of surfactant we used in each case provides approximately monolayer covering to the bare calcite particles providing a degree of steric stabilisation. Now, a finite stress, $\sigma^{*}$, is needed to push them into frictional contact. Thus, $\sigma^{*}$ is a measure of the stress needed to displace adsorbed surfactants from the calcite surface. Once the surfactant is displaced, the frictional interaction is again that between bare particles, accounting for the same $\phi_{\mu}$ in all four suspensions.

In the case of PAA adsorbing on calcite, we can show quantitatively that this is a reasonable suggestion. The adsorption energy of 2000 Da PAA (we used $5100 \mathrm{Da}$ ) on calcite at room temperature is $E=15 \mathrm{~kJ} \mathrm{~mol}^{-1} \approx$ $6 k_{B} T$ per polymer coil (Sparks et al. 2015). This allows us to estimate a local critical stress scale for desorption $\sigma_{0}^{*}=E / R_{g}^{3} \sim 10^{6} \mathrm{~Pa}$, which can be converted into the area of contact when friction is turned on, $r_{0}^{2}$, by equating the bulk and local forces, $\sigma_{0}^{*} r_{0}^{2} \sim \sigma^{*} d^{2}$, for particles of linear size $d$. This gives $r_{0} \sim 7 \mathrm{~nm}$, which is plausibly the length scale of surface roughness; so the picture is that $\sigma^{*}$ is the external stress needed to drive adsorbed PAA from asperities, exposing these to interact frictionally. This highlights the role of surfactant adsorption at the local level, as raised by Mantellato and Flatt (2020) for partial coverage of superplasticiser in cementitious suspensions.

These findings lead to a new design principle for using surfactants to 'tune' the rheology of $\mathrm{nB}$ adhesive suspensions. Given two surfactants that are equally effective as steric stabilisers, i.e. in lowering $\sigma_{a}$, the one that is more strongly adsorbed, i.e. with the higher adsorption energy $E$, should give a higher $\sigma^{*}$ and therefore be more effective in lowering $\sigma_{y}$. Indeed, in the limit of a high enough $\sigma^{*}$ relative to $\sigma_{a}$, adhesive bonds are all broken before any frictional contact network can be formed. The latter can therefore never be stabilised by adhesion, and a yield stress cannot emerge below $\phi_{\mu}$. Thus, $\sigma_{y}$ is reduced from some finite value to zero for all $\phi<\phi_{\mu}$, as is observed when we add PAA or ANS (Table 2). Such use of surfactants to confer a finite onset stress for $\mathrm{nB}$ adhesive suspensions to give essentially an infinite-fold reduction in 
the yield stress is perhaps the main, and certainly the most surprising, conclusion of this work, generalising a similar but less clear-cut finding in the use of surfactants to tune the rheology of molten chocolate (Blanco et al. 2019).

Acknowledgements The authors would like to thank Elena Blanco for providing the SEM micrograph (Fig. 2).

Funding JAR was funded by the UK Engineering and Physical Sciences Research Council (EPSRC) Centre for Doctoral Training in Soft Matter and Functional Interfaces (SOFI CDT) (Grant No. EP/L015536/1), and AkzoNobel, who also provided the surfactants used in this work. WCKP and RO'N were funded by the EPSRC (Grant No. EP/N025318/1).

Data availability The datasets generated during and/or analysed during the current study are available in the Edinburgh DataShare repository, https://doi.org/10.7488/ds/2875.

\section{Compliance with ethical standards}

Conflict of interest The authors declare that they have no competing interests.

Open Access This article is licensed under a Creative Commons Attribution 4.0 International License, which permits use, sharing, adaptation, distribution and reproduction in any medium or format, as long as you give appropriate credit to the original author(s) and the source, provide a link to the Creative Commons licence, and indicate if changes were made. The images or other third party material in this article are included in the article's Creative Commons licence, unless indicated otherwise in a credit line to the material. If material is not included in the article's Creative Commons licence and your intended use is not permitted by statutory regulation or exceeds the permitted use, you will need to obtain permission directly from the copyright holder. To view a copy of this licence, visit http:// creativecommonshorg/licenses/by/4.0/.

\section{Appendix 1. Choosing surfactant concentrations}

Three surfactants, PAA, ANS and PCE, were used in this work to modify the interaction between calcite particles. We determined the concentration used for detailed investigation in each case by measuring the flow curve for a $\phi=$ 0.44 suspension in a $50 \mathrm{wt} . \%$ glycerol-water mixture at increasing surfactant concentration until we reached the point where a further increase does not bring about further changes in the rheological behaviour. Measurements were made under imposed shear rate (TA Instruments ARES-G2, sandblasted plates) with a sweep at 6 points per decade from $\dot{\gamma}_{\min }=0.1 \mathrm{~s}^{-1}$ using a fixed step time of $20 \mathrm{~s}$ equilibration and $10 \mathrm{~s}$ of measurement. Note that as the PCE was supplied in a liquid form, we take the active component to be 50 wt.\% based on the product specification. The addition of surfactants at the concentrations used was found not to affect the glycerol-water mixture viscosities.

The data collected in this process are shown in Fig. 4 for the three surfactants. For all cases we see a saturation in the effect of the surfactant, with no further change in the rheology measured after $0.05 \mathrm{w} / \mathrm{w} \%$ for PAA, $0.5 \mathrm{w} / \mathrm{w} \%$ for ANS and $1 \mathrm{w} / \mathrm{w} \%$ for PCE and the concentration at saturation is used for the experiments reported in the main text. (Note that a single anomalous flow curve was seen for $0.1 \mathrm{w} / \mathrm{w} \%$ PCE (Fig. 4c); this is possibly due to sample under-filling.)

The amount of surfactant needed to achieve saturation effect can be understood quantitatively in the case of the PAA we used, which has a gyration radius of $R_{g} \approx 3 \mathrm{~nm}$. It is easy to estimate that at $0.05 \mathrm{w} / \mathrm{w} \%$, PAA coils of area $\sim R_{g}^{2}$ can cover $\approx 0.5 \mathrm{~m}^{2} / \mathrm{g}$ of calcite surfaces. Separately, modelling our calcite particles as spheres with diameter $d=$ $4 \mu \mathrm{m}$, we estimate that the suspension has a specific surface area of $\approx 0.5 \mathrm{~m}^{2} / \mathrm{g}$, although this only an estimate due to asphericity and polydispersity. The saturation concentration therefore credibly represents complete monolayer coverage. We may surmise that the same may be true for the other two surfactants. This finding lends credence to our suggestion that the finite onset stress conferred by surfactants at the saturation concentration scales with the local stress needed to dislodge adsorbed surfactant molecules.

\section{Appendix 2. Sedimentation tests}

To probe whether bare calcite particles remain adhered after flow, sedimentation tests comparing bare and PAAstabilised particles were carried out on dilute suspensions. Samples of $2 \%$ volume fraction were prepared using
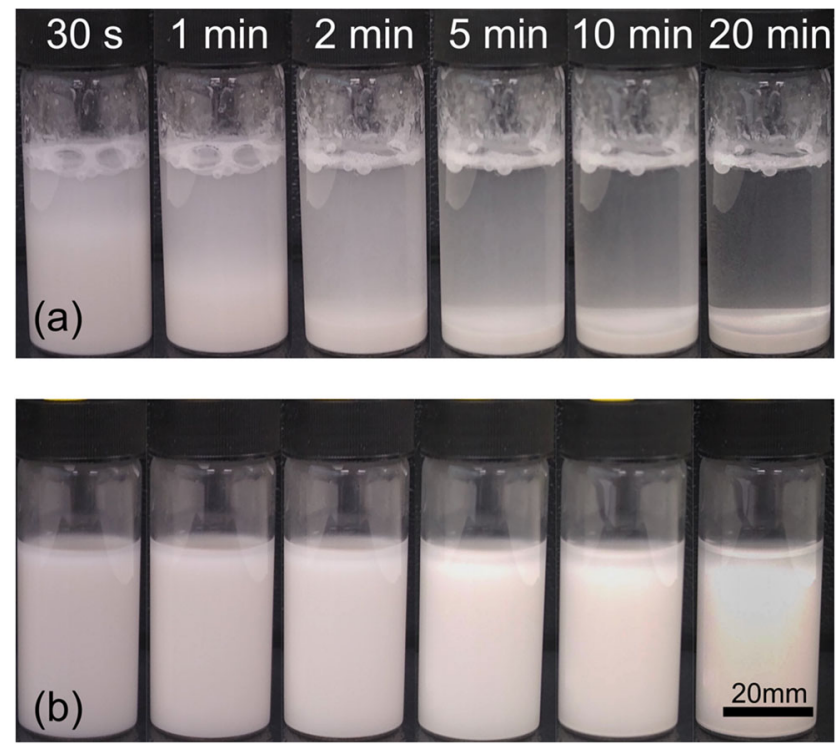

Fig. 8 Sedimentation test of dilute calcite suspensions. a Bare calcite suspension ( $2 \%$ volume fraction in water) sedimentation over time, images taken from $30 \mathrm{~s}$ to $20 \mathrm{~min}$ at indicated times. b PAA-stabilised calcite suspension, as in a but with calcite dispersed with $0.1 \mathrm{w} / \mathrm{w} \%$ PAA. Scale for all images shown bottom right 
distilled water alone and with $0.1 \mathrm{w} / \mathrm{w} \%$ PAA (giving an excess of PAA based on Fig. 4), with the absence of glycerol making sedimentation more rapidly observable. Particles were dispersed using a mixture of vortex and high-shear mixing, followed by 15 min slow mixing on a roller bank. Sedimentation was then monitored though photographing the visible sediment height at the indicated times $(30 \mathrm{~s}$ to $20 \mathrm{~min}$ ), Fig. 8. The bare calcite suspension is observed to sediment more rapidly than the PAA-stabilised suspension (cf. Fig. 8a and b). As the solvent viscosity and density difference are equal, faster sedimentation can be ascribed to larger effective particles. The lack of sedimentation visible in the PAA-stabilised system is consistent with the predicted sedimentation speed for $d \approx 4 \mu \mathrm{m}$ particles, $v=\Delta \rho d^{2} / 18 \eta_{s} \approx 1.5 \mu \mathrm{m} \mathrm{s}^{-1}$, taking $7 \mathrm{~h}$ to sediment $\sim 4 \mathrm{~cm}$. Therefore, bare particles are flocculated and remain adhered in the absence of shear, supporting our conclusions from the rheology at high concentrations.

\section{References}

Al Mahrouqi D, Vinogradov J, Jackson MD (2017) Zeta potential of artificial and natural calcite in aqueous solution. Adv Colloid Interface Sci 240:60-76. https://doi.org/10.1016/j.cis.2016.12.006 Barnes HA (1989) Shear-thickening ("dilatancy") in suspensions of nonaggregating solid particles dispersed in Newtonian liquids. J Rheol 33(2):329-366. https://doi.org/10.1122/1.550017

Bergström L (1997) Hamaker constants of inorganic materials. Adv Colloid Interface Sci 70:125-169. https://doi.org/10.1016/S00018686(97)00003-1

Blanco E, Hodgson DJM, Hermes M, Besseling R, Hunter GL, Chaikin PM, Cates ME, Van Damme I, Poon WCK (2019) Conching chocolate is a prototypical transition from frictionally jammed solid to flowable suspension with maximal solid content. Proc Natl Acad Sci (USA) 116:10303-10308. https://doi.org/10.1073/ pnas. 1901858116

Bonn D, Denn MM, Berthier L, Divoux T, Manneville S (2017) Yield stress materials in soft condensed matter. Rev Mod Phys 89:035005. https://doi.org/10.1103/RevModPhys.89.035005

Bossis G, Boustingorry P, Grasselli Y, Meunier A, Morini R, Zubarev A, Volkova O (2017) Discontinuous shear thickening in the presence of polymers adsorbed on the surface of calcium carbonate particles. Rheol Acta 56(5):415-430. https://doi.org/10.1007/ s00397-017-1005-4

Brown E, Jaeger HM (2014) Shear thickening in concentrated suspensions: phenomenology, mechanisms and relations to jamming. Rep Prog Phys 77(4):046602. https://doi.org/10.1088/0034-4885/ $77 / 4 / 046602$

Brown E, Forman NA, Orellana CS, Zhang H, Maynor BW, Betts DE, DeSimone JM, Jaeger HM (2010) Generality of shear thickening in dense suspensions. Nat Mater 9(3):220. https://doi.org/ $10.1038 /$ nmat 2627

Clavaud C, Bérut A, Metzger B, Forterre Y (2017) Revealing the frictional transition in shear-thickening suspensions. Proc Natl Acad Sci (USA) 114(20):5147-5152. https://doi.org/10.1073/pnas.170 3926114

Comtet J, Chatté G, Niguès A, Bocquet L, Siria A, Colin A (2017) Pairwise frictional profile between particles determines discontinuous shear thickening transition in non-colloidal suspensions. Nat Commun 8:15633. https://doi.org/10.1038/ncomms15633
Dhar S, Chattopadhyay S, Majumdar S (2020) Signature of jamming under steady shear in dense particulate suspensions. J Phys: Condens Matter 32(12):124002. https://doi.org/10.1088/1361-648X/ ab5bd2

Dominik C, Tielens AGGM (1995) Resistance to rolling in the adhesive contact of two elastic spheres. Philosophical Magazine A 72(3):783-803. https://doi.org/10.1080/01418619508243800

Eriksson R, Merta J, Rosenholm JB (2007) The calcite/water interface: I. Surface charge in indifferent electrolyte media and the influence of low-molecular-weight polyelectrolyte. J Colloid Interface Sci 313(1):184-193. https://doi.org/10.1016/j.jcis.2007.04.034

Estrada N, Azéma E, Radjai F, Taboada A (2011) Identification of rolling resistance as a shape parameter in sheared granular media. Phys Rev E 84:011306. https://doi.org/10.1103/PhysRevE. 84.011306

Guy BM, Hermes M, Poon WCK (2015) Towards a unified description of the rheology of hard-particle suspensions. Phys Rev Lett 115(8):088304. https://doi.org/10.1103/PhysRevLett.115.088304

Guy BM, Richards JA, Hodgson DJM, Blanco E, Poon WCK (2018) Constraint-based approach to granular dispersion rheology. Phys Rev Lett 121:128001. https://doi.org/10.1103/PhysRevLett.121. 128001

Heim LO, Blum J, Preuss M, Butt HJ (1999) Adhesion and friction forces between spherical micrometer-sized particles. Phys Rev Lett 83:3328-3331. https://doi.org/10.1103/PhysRevLett.83.3328

Heymann L, Peukert S, Aksel N (2002) On the solid-liquid transition of concentrated suspensions in transient shear flow. Rheol Acta 41(4):307-315. https://doi.org/10.1007/s00397-002-0227-1

Hsu CP, Ramakrishna SN, Zanini M, Spencer ND, Isa L (2018) Roughness-dependent tribology effects on discontinuous shear thickening. Proc Natl Acad Sci USA 115(20):5117-5122. https:// doi.org/10.1073/pnas.1801066115

James NM, Han E, de la Cruz RAL, Jureller J, Jaeger HM (2018) Interparticle hydrogen bonding can elicit shear jamming in dense suspensions. Nat Mater 17(11):965-970. https://doi.org/10.1038/ s41563-018-0175-5

Krieger IM, Dougherty TJ (1959) A mechanism for non-Newtonian flow in suspensions of rigid spheres. Transac Soc Rheol 3:137152. https://doi.org/10.1122/1.548848

KSL Staubtechnik GmbH (2007) Eskal 500 datasheet

Lerner E, Düring G, Wyart M (2012) A unified framework for nonBrownian suspension flows and soft amorphous solids. Proc Natl Acad Sci (USA) 109:4798-4803. https://doi.org/10.1073/pnas.11 20215109

Liberto T, Barentin C, Colombani J, Costa A, Gardini D, Bellotto M, Le Merrer M (2019) Simple ions control the elasticity of calcite gels via interparticle forces. J Colloid Interface Sci 553:280-288. https://doi.org/10.1016/j.jcis.2019.05.083

Lin NYC, Guy BM, Hermes M, Ness C, Sun J, Poon WCK, Cohen I (2015) Hydrodynamic and contact contributions to continuous shear thickening in colloidal suspensions. Phys Rev Lett 115(22):228304. https://doi.org/10.1103/PhysRevLett. 115.228304

Liu W, Jin Y, Chen S, Makse HA, Li S (2017) Equation of state for random sphere packings with arbitrary adhesion and friction. Soft Matter 13:421-427. https://doi.org/10.1039/C6SM02216B

Mantellato S, Flatt RJ (2020) Shifting factor-A new paradigm for studying the rheology of cementitious suspensions. J Am Ceram Soc 103(6):3562-3574. https://doi.org/10.1111/jace.17040

Mechtcherine V, Bos F, Perrot A, da Silva WL, Nerella V, Fataei S, Wolfs R, Sonebi M, Roussel N (2020) Extrusion-based additive manufacturing with cement-based materials - Production steps, processes, and their underlying physics: A review. Cem Concr Res 132:106037. https://doi.org/10.1016/j.cemconres.2020.106037

Mehta PK (1999) Advancements in concrete technology. Concr Int 21(6):69-76 
Piotte M, Bossányi F, Perreault F, Jolicoeur C (1995) Characterization of poly(naphthalenesulfonate) salts by ion-pair chromatography and ultrafiltration. J Chromatogr A 704(2):377-385. https://doi.org/10.1016/0021-9673(95)00226-D

Reith D, Müller B, Müller-Plathe F, Wiegand S (2002) How does the chain extension of poly (acrylic acid) scale in aqueous solution? a combined study with light scattering and computer simulation. J Chem Phys 116(20):9100-9106. https://doi.org/10.1063/ 1.1471901

Richards JA, Guy BM, Blanco E, Hermes M, Poy G, Poon WCK (2020) The role of friction in the yielding of adhesive nonBrownian suspensions. J Rheol 64(2):405-412. https://doi.org/ $10.1122 / 1.5132395$

Roussel N (2007) Rheology of fresh concrete: from measurements to predictions of casting processes. Mater Struct 40(10):10011012. https://doi.org/10.1617/s11527-007-9313-2

Seto R, Mari R, Morris JF, Denn MM (2013) Discontinuous shear thickening of frictional hard-sphere suspensions. Phys Rev Lett 111:218301. https://doi.org/10.1103/PhysRevLett.111.218301

Silbert LE (2010) Jamming of frictional spheres and random loose packing. Soft Matter 6(13):2918-2924. https://doi.org/10.1039/ C001973A
Sparks DJ, Romero-González ME, El-Taboni E, Freeman CL, Hall SA, Kakonyi G, Swanson L, Banwart SA, Harding JH (2015) Adsorption of poly acrylic acid onto the surface of calcite: an experimental and simulation study. Phys Chem Chem Phys 17:27357-27365. https://doi.org/10.1039/C5CP00945F

Wildemuth CR, Williams MC (1985) A new interpretation of viscosity and yield stress in dense slurries: Coal and other irregular particles. Rheol Acta 24(1):75-91. https://doi.org/10.1007/BF01329266

Wilson HJ, Davis RH (2000) The viscosity of a dilute suspension of rough spheres. J Fluid Mech 421:339-367. https://doi.org/ 10.1017/S0022112000001695

Wyart M, Cates ME (2014) Discontinuous shear thickening without inertia in dense non-Brownian suspensions. Phys Rev Lett 112(9):098302. https://doi.org/10.1103/PhysRevLett.112.098302

Zhou JZQ, Uhlherr PHT, Luo FT (1995) Yield stress and maximum packing fraction of concentrated suspensions. Rheol Acta 34(6):544-561. https://doi.org/10.1007/BF00712315

Publisher's note Springer Nature remains neutral with regard to jurisdictional claims in published maps and institutional affiliations. 